\title{
Método dos Mínimos Quadrados Complexos: Aplicações ao Estudo por Impedância Eletroquímica da Corrosão em Ligas de Titânio
}

J.M.V. CAPELA ${ }^{1}$, M.V. CAPELA, R. MAGNANI, Área de Matemática do Departamento de Físico-Química, IQ, UNESP, 14800-900 Araraquara, SP, Brasil.

Resumo. O método dos Mínimos Quadrados Complexos é utilizado para a estimação de parâmetros de impedância eletroquímica. Este método pressupõe que as partes real e imaginária da impedância sejam independentes, o que em geral não é verdadeiro em experimentos eletroquímicos. O objetivo deste trabalho é apresentar um critério de estimação de parâmetros mais geral, de tal forma que a correlação entre as partes real e imaginária da impedância não seja desprezada. Além disso, procurou-se estabelecer um modelo de regressão adequado à impedância de um sistema eletroquímico experimental constituído por uma liga metálica de Titânio imersa em uma solução de cloreto de sódio a $0,9 \%$.

\section{Introdução}

A espectroscopia de impedância eletroquímica é uma técnica importante para o estudo de reações eletroquímicas que ocorrem em sistemas físicos, químicos ou biológicos. O procedimento experimental consiste na observação da resposta do sistema em estudo, quando perturbado por um sinal de teste senoidal com amplitude suficientemente pequena e freqüência angular variando. A condição de amplitude pequena é necessária para que o sistema seja linear e, portanto, com o seu comportamento dinâmico descrito por uma equação diferencial linear [4], [6].

A impedância de um sistema eletroquímico linear é definida como sendo o quociente entre a transformada de Laplace da resposta e a transformada de Laplace da perturbação do sistema [6]. Trata-se de uma função de variável complexa da forma

$$
f(w, \Theta)=f_{1}(w, \Theta)+i f_{2}(w, \Theta)
$$

onde $i^{2}=-1$ é a unidade imaginária, $\Theta$ é um vetor de parâmetros a ser estimado e $w$ é a frequência angular.

Em geral, a função (1.1) é obtida de um circuito elétrico com impedância equivalente à do sistema original, com a condição de que cada elemento do circuito possua significado físico apropriado.

\footnotetext{
${ }^{1}$ capela@iq.unesp.br
} 
Após determinar a função complexa de impedância procura-se um modelo de regressão adequado ao sistema em estudo. Tal modelo de regressão é uma relação entre as $n$ variáveis aleatórias complexas de impedância, $z_{k}=z_{k 1}+i z_{k 2}, k=1, \ldots, n$ e os $n$ valores fixos da frequência angular $w_{k}$.

Neste trabalho supõe-se que o modelo de regressão seja da forma

$$
z_{k}=f\left(w_{k}, \Theta\right)+\epsilon_{k}
$$

sendo $f\left(w_{k}, \Theta\right)$ a função complexa de impedância, também denominada função de regressão e $\epsilon_{k}=\epsilon_{k 1}+i \epsilon_{k 2}$ um erro aleatório, ou uma perturbação no valor esperado da variável $z_{k}$.

Um dos objetivos da análise de um sistema eletroquímico por espectroscopia de impedância eletroquímica é a estimação do vetor de parâmetros $\Theta$. No caso dos estudos de corrosão o conhecimento das estimativas desses parâmetros permite prever o comportamento de um determinado material em um meio corrosivo agressivo.

$\mathrm{O}$ vetor de estimativas dos parâmetros é obtido com base num conjunto de valores experimentais, sendo que usualmente são determinadas como estimativas os valores que produzem a menor soma de quadrados dos desvios das partes real e imaginária das observações experimentais em relação à função de regressão [2], [7]. Esse critério de estimação, denominado método dos Mínimos Quadrados Complexos (MQC), equivale à minimização do traço da matriz de covariâncias dos erros aleatórios $\epsilon_{k 1}$ e $\epsilon_{k 2}, k=1,2, \ldots, n$, e pressupõe que os mesmos tenham um comportamento probabilístico normal de média zero, variância constante e sejam independentes [1].

Neste trabalho apresenta-se um critério de estimação mais geral, que considere, ao contrário do método dos Mínimos Quadrados Complexos, a correlação entre os erros reais e imaginários. Esse critério é aqui denominado de método dos Mínimos Quadrados Complexos Generalizados (MQCG).

Os resultados foram aplicados com o objetivo de estabelecer um modelo de regressão adequado e determinar as estimativas dos parâmetros de impedância de um sistema eletroquímico constituído por uma liga de titânio imersa em uma solução de cloreto de sódio . Sistemas eletroquímicos desta natureza são importantes em estudos sobre a resistência do Titânio à corrosão em meios que simulam a agressividade corrosiva dos tecidos do corpo.

\section{Metodologia}

O vetor de estimativas dos parâmetros, $\widehat{\Theta}$, será obtido supondo-se que os termos aleatórios, $\epsilon_{k m}, k=1, \ldots, n ; m=1,2$, tenham média zero e covariâncias conhecidas, dadas por

$$
\operatorname{cov}\left(\epsilon_{k m}, \epsilon_{r s}\right)= \begin{cases}\Sigma_{m s}, & \text { se } k=r, \\ 0, & \text { se } k \neq r,\end{cases}
$$

para $k, r=1, \ldots, n$ e $m, s=1,2$.

A estimativa de máxima verossimilhança dos parâmetros é o vetor $\widehat{\Theta}$ de uma população hipotética que gera a máxima verossimilhança para os valores da amostra 
$z_{k}$. A verossimilhança de tal população haver gerado a amostra extraída é dada pela densidade de probabilidade conjunta dos $z_{k}$, expressa como uma função dos possíveis valores dos parâmetros e das covariâncias da população.

Supondo que a distribuição de probabilidade dos $z_{k}$ seja normal, tem-se a seguinte função de verossimilhança da amostra [1]

$$
L\left(\Theta, U^{-1}\right)=\frac{\left|U^{-1}\right|^{\frac{n}{2}}}{2 \pi^{\frac{n}{2}}} e^{-\left[\frac{t r\left(E U^{-1} E^{t}\right)}{2}\right]}
$$

sendo $U^{-1}=\left(\Sigma_{m s}^{*}\right)_{2 \times 2}$ a inversa da matriz de covariâncias e $E=\left(\epsilon_{k m}\right)_{n \times 2}$ a matriz dos erros aleatórios. As barras denotam o determinante e $t r$ o traço da matriz.

Derivando-se o logaritmo da equação (2.2) com relação a $\Sigma_{m s}^{*}$, obtém-se a seguinte expressão

$$
\frac{\partial \ln L\left(\Theta, U^{-1}\right)}{\partial \Sigma_{m s}^{*}}=\frac{n}{2} \Sigma_{m s}-\frac{1}{2}\left\{E^{t} E\right\}_{m s} .
$$

Igualando-se esta derivada a zero, encontra-se que a estimativa da matriz de covariâncias é dada por

$$
\widehat{U}=\frac{E^{t} E}{n}
$$

Portanto, substituindo-se essa estimativa $\widehat{U}$ no logaritmo da equação (2.2), tem-se

$$
\ln L(\Theta, \widehat{U})=\ln \left(\frac{1}{(2 \pi n)^{\frac{n}{2}}}\right)-n-\frac{n}{2} \ln \left|E^{t} E\right| .
$$

O máximo de $\ln L(\Theta, \widehat{U})$, que corresponde ao máximo de $L(\Theta, \widehat{U})$, é obtido pela minimização do determinante $\left|E^{t} E\right|$ da equação (2.4).

As estimativas dos parâmetros que produzem o valor mínimo do determinante $\left|E^{t} E\right|$ são chamadas estimativas de Mínimos Quadrados Complexos Generalizados (MQCG).

Se os erros reais e imaginários forem independentes, então

$$
\left\{E^{t} E\right\}_{12}=\left\{E^{t} E\right\}_{21}=0,
$$

e o termo $\ln \left|E^{t} E\right|$ da equação (2.4) pode ser escrito como

$$
\begin{aligned}
\ln \left|E^{t} E\right| & =\ln \left(\left\{E^{t} E\right\}_{11}\left\{E^{t} E\right\}_{22}\right) \\
& =\ln \left(\left\{E^{t} E\right\}_{11}\right)+\ln \left(\left\{E^{t} E\right\}_{22}\right)
\end{aligned}
$$

e a minimização de $\ln \left|E^{t} E\right|$ é equivalente à minimização do traço

$$
\operatorname{tr}\left(E^{t} E\right)=\left\{E^{t} E\right\}_{11}+\left\{E^{t} E\right\}_{22} .
$$

Este critério é conhecido como método dos Mínimos Quadrados Complexos (MQC). Um intervalo de $100(1-\alpha) \%$ de confiança para o parâmetro $\theta_{k}, k=1,2, \ldots, p$, pode ser determinado por

$$
\widehat{\theta}_{k} \pm t(n-p ; \alpha / 2) s \sqrt{2\left\{\Omega^{-1}\right\}_{k k}}
$$


sendo $\left\{\Omega^{-1}\right\}_{k k}$ o $k$-ésimo elemento da diagonal da inversa da matriz hessiana, $t(n-p ; \alpha / 2)$ o valor da distribuição $t$ de Student com $n-p$ graus de liberdade e $s^{2}=\left|E^{t}(\widehat{\Theta}) E(\widehat{\Theta})\right| /(n-p)$ uma estimativa da variância baseada em $n-p$ graus de liberdade [1].

\section{Resultados e Discussão}

A função que descreve a impedância do sistema eletroquímico experimental constituído por uma liga metálica de Titânio imersa em uma solução de cloreto de sódio a $0,9 \%$, é dada por $([3],[5])$

$$
f(w, \Theta)=\frac{a}{(1-i w b)^{\alpha}}
$$

sendo $\Theta=(a, b, \alpha)^{T}$ é o vetor de parâmetros, $w$ a freqüência angular e $i^{2}=-1$ a unidade imaginária. Os parâmetros $a$ e $b$ envolvem resistências (em ohms, $\Omega$ ) e capacitâncias (em farads, $F$ ) do sistema. O parâmetro $\alpha$ é útil para se comparar o comportamento da impedância de uma interface eletroquímica (região interfacial entre o metal e a solução eletrolítica) qualquer com a impedância de uma interface na qual a superfície do metal é plana, correspondendo a $\alpha=1$.

Os resultados das estimativas de Mínimos Quadrados Complexos Generalizados (MQCG) e Mínimos Quadrados Complexos (MQC) dos parâmetros e a amplitude de seus respectivos intervalos de confiança, encontram-se na Tabela 1. A representação no plano complexo dos valores experimentais da impedância e dos valores calculados com essas estimativas é dada na Figura 1a, onde a seta indica o sentido crescente dos valores da freqüência $w$. Na Figura 1 b estão representados, no plano complexo, os erros residuais $\epsilon_{k 1}$ e $\epsilon_{k 2}, k=1,2, \ldots, n$.

Observa-se na Tabela 1 que as estimativas pontuais dos parâmetros, obtidas com os métodos MQCG e MQC, são aproximadamente iguais. A diferença dos resultados encontra-se somente na amplitude dos intervalos de confiança, sendo que para o método MQCG os intervalos são mais estreitos. Nota-se também, na Figura $1 b$, que os erros residuais reais e imaginários não se encontram aleatoriamente distribuídos em torno da origem do plano complexo. Este fato indica que eles estão correlacionados e as restrições $\operatorname{cov}\left(\epsilon_{k 1}, \epsilon_{k 2}\right)=\operatorname{cov}\left(\epsilon_{k 2}, \epsilon_{k 1}\right)=0$ não são válidas. Assim, os intervalos de estimação para os MQC podem estar centrados em estimativas inexatas dos parâmetros, sendo este erro compensado com intervalos mais amplos.

Nas figuras 2a e 2b observa-se que os erros residuais apresentam correlação serial (ou autocorrelação), isto é, fixado um valor de $k$, o erro $\epsilon_{k+1}$ encontra-se correlacionado com o valor prévio $\epsilon_{k}$. Como a autocorrelação aparenta ser linear, é possível supor que a seguinte relação é válida

$$
\epsilon_{k+1, m}=\epsilon_{k+1, m}^{*}+\delta_{m} \epsilon_{k, m},
$$

onde $\epsilon_{k+1, m}^{*}, \quad k=1,2, \cdots, N ; \quad m=1,2$, são erros aleatórios independentes de média zero e variância constante e $\delta_{m}$ um parâmetro a ser determinado. 
Tabela 1: Intervalos de confiança para as estimativas de MQCG e MQC dos parâmetros $a, b$ e $\alpha$ da impedância dada pela função (3.1).

\begin{tabular}{lccc}
\hline Método & $a / \Omega$ & $b / \Omega F$ & $\alpha$ \\
\hline MQCG & $6,7 \times 10^{6} \pm 2,1 \times 10^{4}$ & $169,1 \pm 0,9$ & $0,9 \pm 8,4 \times 10^{-4}$ \\
MQC & $6,7 \times 10^{6} \pm 3,4 \times 10^{4}$ & $169,4 \pm 1,31$ & $0,9 \pm 1,2 \times 10^{-3}$ \\
\hline
\end{tabular}
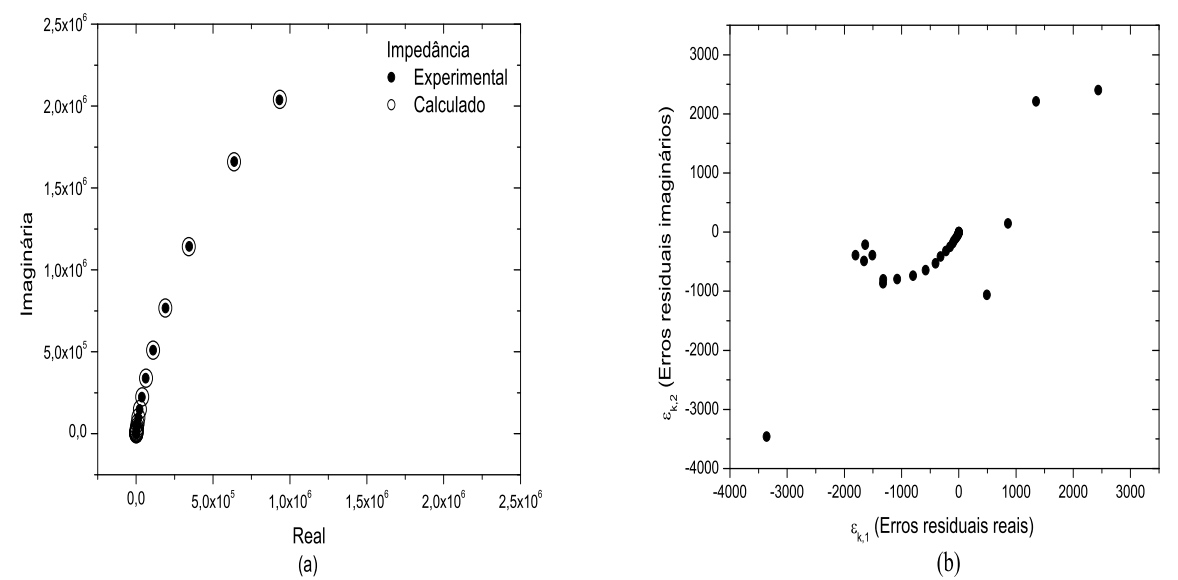

(b)

Figura 1: Representação no plano complexo (a) dos valores experimentais e dos valores calculados da impedância com as estimativas de MQCG dos parâmetros, (b) dos valores dos erros residuais correspondentes.

A substituição do processo autoregressivo dado pela equação (3.2) na relação (1.2) resulta em

$$
\begin{aligned}
z_{k+1}= & f\left(w_{k+1}, \Theta\right) \\
& +\delta\left(z_{k}-f\left(w_{k}, \Theta\right)\right)+\epsilon_{k+1}^{*},
\end{aligned}
$$

onde $k=1,2, \cdots, N, \epsilon_{k+1}^{*}=\epsilon_{k+1,1}^{*}+i \epsilon_{k+1,2}^{*}$, e $\delta=\delta_{1}+i \delta_{2}$.

As estimativas de Mínimos Quadrados Complexos Generalizados e de Mínimos Quadrados Complexos dos parâmetros, obtidas quando se considera o processo autoregressivo dado pela equação (3.3), estão na Tabela 2.

Com o objetivo de avaliar a qualidade dos ajustes realizados considerou-se nas figuras 3a e 3b, respectivamente, a representação gráfica dos erros residuais relativos para o caso do modelo de regressão dado pela equação (1.2) e para o caso do modelo 


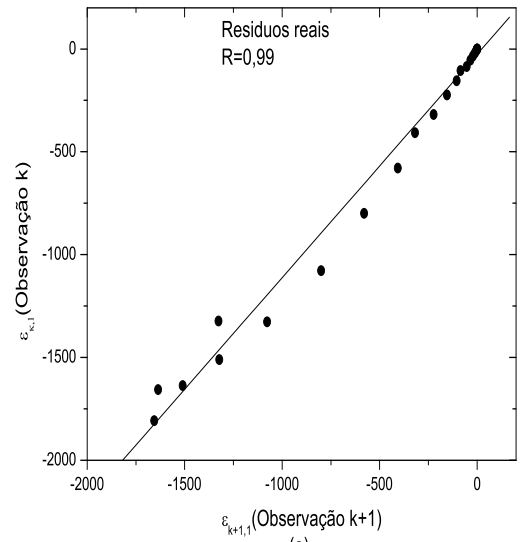

(a)

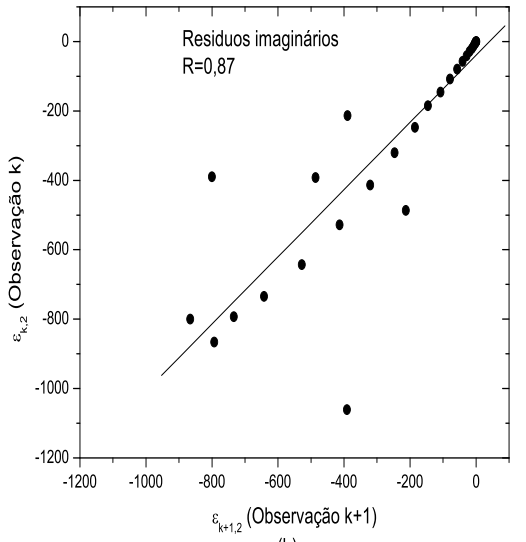

(b)

Figura 2: Correlação serial entre os erro residuais, (a) erros reais (b) erros imaginários.

Tabela 2: Estimativas de MQCG e MQC dos parâmetros de impedância $a, b$ e $\alpha$ quando se considera o processo autoregressivo de primeira ordem e a relação (3.3).

\begin{tabular}{lccccc}
\hline Método & $a / \Omega$ & $b / \Omega F$ & $\alpha$ & $\delta_{1}$ & $\delta_{2}$ \\
\hline MQCG & $7,1 \times 10^{6}$ & 177,8 & 0,89 & 0,76 & 0,75 \\
MQC & $7,0 \times 10^{6}$ & 176,5 & 0,89 & 0,76 & 0,75 \\
\hline
\end{tabular}

autoregressivo, dado pela equação (3.3).

Os erros residuais relativos são definidos por

$$
E_{k m}=\left|\frac{z_{k m}^{\text {exper. }}-f_{m}\left(w_{k}, \widehat{\Theta}\right)}{z_{k m}^{\text {exper. }}}\right|,
$$

sendo $k=1,2, \ldots, n$ e $m=1,2$. Os termos $z_{k m}^{\text {exper. }}$ e $f_{m}\left(w_{k}, \widehat{\Theta}\right)$ representam, respectivamente, os valores experimentais e os valores calculados da impedância para o vetor de estimativas $\widehat{\Theta}$.

Comparando-se os resultados das figuras $3 \mathrm{a}$ e $3 \mathrm{~b}$ verifica-se que para o modelo autoregressivo os erros relativos foram menores. Os resultados da Tabela 2 mostram que, também neste caso, os métodos MQCG e MQC forneceram estimativas pontuais dos parâmetros aproximadamente iguais. 


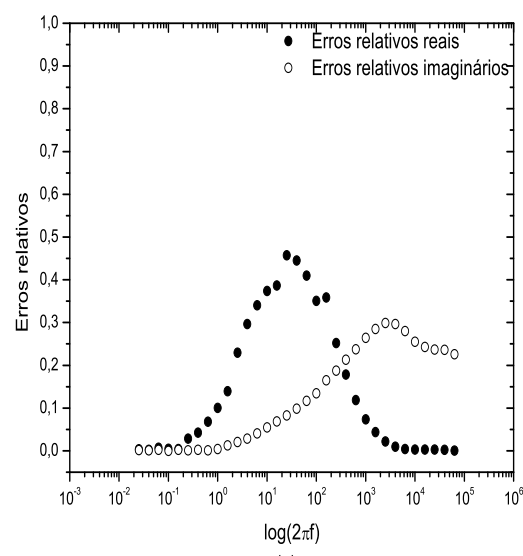

(a)

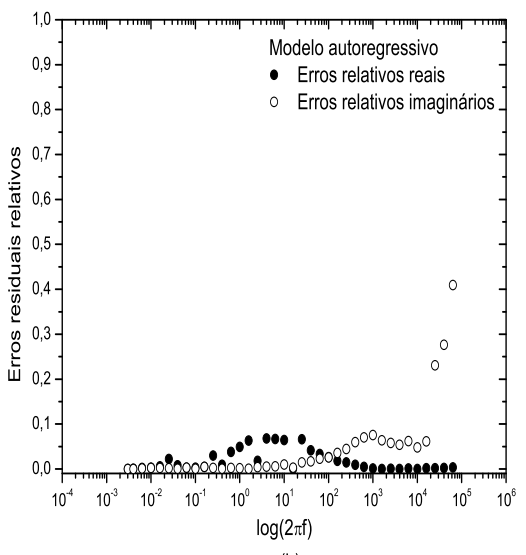

(b)

Figura 3: Erros residuais relativos, (a) modelo de regressão dado pela equação (1.2) (b) modelo autoregressivo, dado pela equação (3.3).

\section{Conclusões}

O método dos Mínimos Quadrados Complexos Generalizados é teoricamente mais eficiente que o Método dos Mínimos Quadrados Complexos, por fornecer um intervalo de confiança válido. Entretanto os resultados das estimativas pontuais dos parâmetros por esses dois métodos de estimação foram semelhantes para a situação experimental considerada.

Além disso, pode-se concluir também que a qualidade do ajuste realizado melhorou quando foi adotado um modelo de regressão que levasse em conta a autocorrelação linear entre os erros residuais.

Abstract. The complex least square method is used for the estimation of electrochemical impedance parameters. With this method one supposes that the real and imaginary impedance parts are independent, what is generally not true for electrochemical experiments. In this work, a more general parameters estimation criteria is presented, in a way that the correlation between the real and imaginary impedance parts is not dismissed. Besides, it was tried to establish a regression model suitable for the impedance of an experimental electrochemical system with a $\mathrm{Ti}$ alloy immersed in a $0.9 \% \mathrm{NaCl}$ solution.

\section{Referências}

[1] D.M. Bates, D.G. Watts, "Nonlinear Regression Analysis and its Applications", John Wiley, New York, 1988.

[2] B.A. Boukamp, A nonlinear least squares fit procedure for analysis of immitance data of electrochemical systems. Solid State Ionics, 20 (1986) 31-44. 
[3] J.M.V. Capela, M.V. Capela, R. Magnani, Generalização de um modelo linear de impedância eletroquímica. Eclet. Quim., 28 (2003) 63-67.

[4] R.C. Dorf, R.H. Bishop, "Sistemas de Controle Moderno", LTC, Rio de Janeiro, 2001.

[5] R. De Levie, On impedance of electrodes with rough interfaces. J. Electroanal. Chem., 261 (1989) 1-9.

[6] D.D. Macdonald, E. Sikora, G. Engelhardt, Characterizing electrochemical systems in the frequency domain. Electrochim. Acta, 43 (1998) 87-107.

[7] J.R. Macdonald, J. Schoonman,P.A. Lehnen, The applicability and power of complex nonlinear least squares for the analysis of impedance and admittance data. J. Electroanal. Chem., 131 (1982) 77-95. 